\title{
Análise viscoelástica de pavimentos asfálticos utilizando elementos finitos e infinitos
}

\author{
Samuel Almeida Torquato Silva ${ }^{1}$, Pedro Jose Furlani Vidal ${ }^{2}$, \\ Áurea Silva Holanda ${ }^{3}$ e Evandro Parente Junior ${ }^{4}$
}

\begin{abstract}
Resumo: Este trabalho apresenta uma estratégia para aumentar a precisão e a eficiência computacional da análise de pavimentos flexíveis baseada no uso de elementos infinitos. A geração da malha mista, composta de elementos finitos e infinitos, é realizada de forma a garantir a qualidade dos resultados. Os valores de deflexões e tensões obtidos são comparados com os encontrados na literatura e os resultados de malhas de elementos finitos são comparados, quanto à eficiência computacional e precisão, com os encontrados a partir de malhas mistas. Os exemplos numéricos atestam que os elementos infinitos melhoram tanto a eficiência da análise quanto a precisão das respostas mecânicas calculadas. Portanto, esta abordagem é fortemente recomendada para a utilização prática na análise e projeto de pavimentos flexíveis.

DOI:10.4237/transportes.v21i3.676.
\end{abstract}

Palavras-chave: pavimentos flexíveis, viscoelasticidade linear, método dos elementos finitos.

\begin{abstract}
This paper presents a strategy to increase the accuracy and computational efficiency of the flexible pavement analysis based on the use of infinite elements. The mixed mesh generation, composed of finite and infinite elements, is performed in order to guarantee accurate results. The vertical displacement and stresses are calculated and the results are compared with the ones found in the literature. The computational efficiency and the accuracy of the results are compared when conventional and mixed meshes are adopted. The numerical examples show that the use of infinite elements improve the computational efficiency and the accuracy of the results. Thus, this approach is strongly recommended for practical use in the analysis and design of flexible pavements.
\end{abstract}

Keywords: flexible paviments, linear viscoelasticity, finite element method.

\section{INTRODUÇÃO}

Segundo Bernucci et al. (2007), "pavimento é uma estrutura de múltiplas camadas de espessuras finitas, construída sobre a superfície final de terraplanagem, destinada técnica e economicamente a resistir aos esforços oriundos do tráfego de veículos e do clima e a propiciar aos usuários melhoria nas condições de rolamento, com conforto, economia e segurança".

Os pavimentos podem ser classificados basicamente em rígidos e flexíveis. Os pavimentos rígidos apresentam como revestimento uma placa de concreto de cimento Portland. Esta placa pode ser armada ou não com barras de aço e sua resistência à flexão, juntamente com as resistências das camadas subjacentes, determina a espessura do pavimento. Neste tipo de pavimento a subcamada é normalmente denominada sub-base em função da semelhança da qualidade do material com o utilizado nas sub-bases de pavimentos asfálticos.

Os pavimentos asfálticos são formados de uma maneira geral por quatro camadas principais: revestimento asfáltico, base, sub-base e reforço do subleito. Contudo, algumas des-

\footnotetext{
${ }^{1}$ Samuel Almeida Torquato Silva, Departamento de Engenharia de Transportes, Universidade Federal do Ceará, Fortaleza, CE, Brasil. (e-mail: samuel_almeida90@hotmail.com)

${ }^{2}$ Pedro Jose Furlani Vidal, Departamento de Engenharia de Transportes, Universidade Federal do Ceará, Fortaleza, CE, Brasil. (e-mail: pedrofurlani@hotmail.com)

${ }^{3}$ Áurea Silva Holanda Departamento de Engenharia de Transportes, Universidade Federal do Ceará, Fortaleza, CE, Brasil. (e-mail: aurea@det.ufc.br)

${ }^{4}$ Evandro Parente Junior, Departamento de Engenharia Estrutural e Construção Civil, Universidade Federal do Ceará, Fortaleza, CE, Brasil. (e-mail: evandro@ufc.br)
}

Manuscrito recebido em 22/04/2013 e aprovado para publicação em 24/10/2013. Este artigo é parte de TRANSPORTES v. 21, n. 3, 2013. ISSN: 2237-1346 (online). DOI:10.4237/transportes.v21i3.676. tas camadas podem ser suprimidas em função do tráfego e dos materiais disponíveis na região. O revestimento é formado basicamente de uma mistura de agregados e ligantes asfálticos e pode ser composto por camada de rolamento (em contato direto com os veículos) e camadas intermediárias (binder). O revestimento tem a função de resistir, de forma direta, ao tráfego, atenuando o efeito do mesmo nas camadas inferiores, além de impermeabilizar o pavimento e melhorar as condições de rolamento (Bernucci et al., 2007).

Durante as primeiras décadas do século XX o projeto de pavimentos era marcado por um caráter empírico, ou seja, baseado apenas na experiência dos projetistas. No entanto, com o crescimento econômico e consequente aumento do volume de tráfego e peso dos carregamentos, houve a necessidade de melhorar a qualidade dos pavimentos, bem como o entendimento com relação ao seu comportamento mecânico. Assim, a tendência voltou-se para o uso dos métodos mecanísticos-empíricos nas diversas atividades envolvidas no projeto de pavimentos (Motta, 1991; Medina e Motta, 2005; Huang, 2004; NCHRP/TRB, 2004; Franco, 2007; Soares et al., 2009).

A partir desta nova filosofia, os materiais são previamente caracterizados em laboratório e os resultados obtidos são utilizados como dados de entrada para realização das análises. Estas análises geram valores de tensão e deformação nos pavimentos que são comparados com critérios de dimensionamento pré-definidos, estabelecidos a partir de experiências de campo. É por este motivo que os métodos são normalmente denominados mecanístico-empíricos.

Os materiais que compõem o pavimento flexível apresentam comportamento complexo e é um grande desafio representá-los por modelos que se aproximem da realidade. Existem vários modelos constitutivos que permitem representar a dependência do tempo e a taxa de aplicação do carrega- 
mento observado em misturas asfálticas. O modelo viscoelástico linear, por exemplo, tem sido bastante utilizado na caracterização de misturas asfálticas (Huang, 2004) devido à boa correlação obtida entre a teoria e os ensaios de laboratório (Soares e Souza, 2002). Uma grande vantagem desse modelo é a consideração do tempo de carregamento, pois responde tanto a cargas rápidas quanto a cargas lentas.

Com o objetivo de modelar de forma mais adequada o comportamento dos materiais utilizados em pavimentação, é necessário o uso de métodos numéricos, sendo o Método dos Elementos Finitos (Cook et al., 2002) muito utilizado na análise de pavimentos (NCHRP/TRB, 2004). Uma das grandes preocupações é tornar o programa de baixo custo computacional, sem, contudo, alterar a boa qualidade dos resultados.

O problema de análise de pavimentos apresenta uma característica peculiar que é a necessidade de simular o "comprimento infinito" de seu subleito. Neste caso, a maneira tradicional de resolver este problema, através do truncamento do modelo a uma grande distância da superfície e da região de aplicação da carga, causa um grande aumento no esforço computacional. Por outro lado, um truncamento a uma distância pequena pode acarretar grandes erros nos cálculos dos deslocamentos no topo do revestimento.

Neste trabalho, foram utilizados elementos infinitos (Bettess, 1992) para modelagem do subleito, diminuindo o esforço computacional e melhorando a qualidade dos resultados. Exemplos numéricos serão apresentados com o objetivo de avaliar esta alternativa quanto à eficiência computacional e qualidade nos resultados, tanto para modelos elásticos quanto viscoelásticos.

\section{ANÁLISE VISCOELÁSTICA LINEAR}

O comportamento viscoelástico pode ser interpretado como uma composição das características de um sólido elástico e um fluido viscoso. A deformação de um fluido viscoso depende do tempo $(t)$ e da taxa de aplicação do carregamento. Desta forma, tem-se que o material viscoelástico, quando carregado, possui uma parcela de resposta imediata e outra dependente do tempo.

Sendo materiais elásticos representados por molas e materiais viscosos por amortecedores, tem-se que o material viscoelástico pode ser representado por combinações de molas e amortecedores. Para a representação do revestimento asfáltico, o Modelo de Weichert ou Modelo de Maxwell Generalizado proporciona resultados próximos aos reais quando ajustado com base em resultados de laboratório (Huang, 2004). Este modelo, ilustrado na Figura 1, é formado por uma mola de rigidez $E_{\infty}$ combinada em paralelo com elementos de Maxwell formados por molas de rigidez $E_{i}$ e amortecedores de constante $\eta_{i}$.

A caracterização do material realizada pela aplicação de

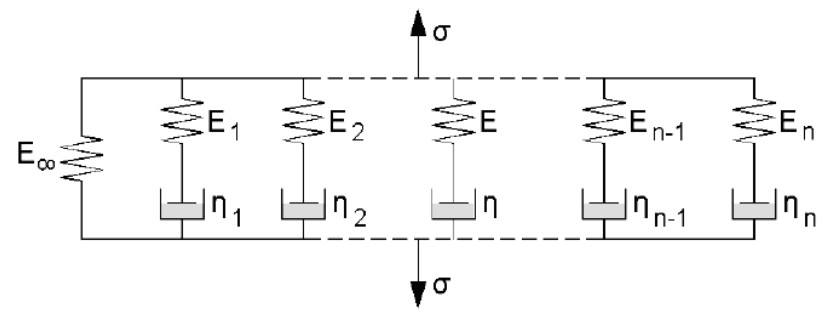

Figura 1. Modelo de Wiechert tensão constante $\left(\sigma_{0}\right)$ e medição da deformação resultante $\varepsilon(t)$ é chamada de ensaio de fluência (creep), enquanto a caracterização pela aplicação de deformação constante $\left(\varepsilon_{0}\right)$ e medição da tensão resultante $\sigma(t)$ é chamada de ensaio de relaxação (relaxation) (Lakes, 1998). São obtidos, destes ensaios, os parâmetros compliância $D(t)$ :

$$
D(t)=\frac{\varepsilon(t)}{\sigma_{0}}
$$

e módulo de relaxação E(t):

$$
E(t)=\frac{\sigma(t)}{\varepsilon_{0}}
$$

Pode-se mostrar (Christensen, 1982; Lakes, 1998) que o módulo de relaxação do modelo de Wiechert pode ser escrito como uma série de Prony-Dirichlet:

$$
E(t)=E_{\infty}+\sum_{i=1}^{n} E_{i} e^{-t / \rho_{i}}
$$

em que $E_{\infty}, E_{i}$ e $\rho_{i}=\eta_{i} / E_{i}$ são os coeficientes a serem obtidos a partir dos ensaios e $n$ é o número de termos da série (correspondentes ao número de elementos de Maxwell da Figura 1).

Como os materiais viscoelásticos apresentam comportamento dependente do tempo e da taxa de aplicação do carregamento, suas respostas mecânicas em um dado instante de tempo t não dependem apenas da carga aplicada neste instante, como no caso dos materiais elásticos, mas de toda a história do carregamento até este instante. Utilizando o Princípio da Superposição de Boltzmann (Christensen, 1982; Lakes, 1998), a relação tensão-deformação de materiais viscoelásticos pode ser escrita como uma integral hereditária:

$$
\sigma(t)=\int_{0}^{t} E(t-\tau) \frac{\partial \varepsilon}{\partial \tau} d \tau
$$

em que $\tau$ é uma variável de integração auxiliar que varia entre 0 e $t$. Esta integral é também conhecida como integral de convolução ou de Volterra.

A análise de sólidos viscoelásticos pode ser realizada através de métodos analíticos ou computacionais. As soluções analíticas normalmente são obtidas utilizando o Princípio da Correspondência Elástica-Viscoelástica, onde a variável tempo é eliminada através do uso da Transformada de Laplace (Shames e Cozzarelli, 1997). Contudo, esta abordagem é restrita a problemas com geometrias, carregamentos e condições de contorno simples. A análise de problemas complexos requer a utilização de métodos numéricos.

A análise pelo Método dos Elementos Finitos (MEF) é realizada através de algoritmos incrementais, onde o tempo é discretizado em uma sequência de passos $t_{0}, \ldots, t_{n}, t_{n+1}$ calculados de forma sequencial:

$$
t_{n+1}=t_{n}+\Delta t
$$

em que $\Delta t$ é o incremento tempo. As tensões são avaliadas apenas nestes instantes discretos:

$$
\sigma_{n+1}=\sigma_{n}+\Delta \sigma
$$


O incremento de tensões $\Delta \sigma$ é calculado integrando-se a Equação (4) entre $t_{n}$ e $t_{n+1}$ utilizando o módulo de relaxação definido pela Equação (3) e considerando que o incremento de deformações $\Delta \varepsilon$ é pequeno no intervalo de tempo considerado. O algoritmo completo para análise estática é apresentado em (Portela et al., 2008) e para análise dinâmica em (Araújo et al., 2010).

\section{ELEMENTOS INFINITOS}

O uso de elementos infinitos tem como motivação a solução de diversos problemas cuja região de interesse não possui condições de contorno bem definidas, tais como aplicação de carga em meios semi-infinitos, fluidos e campos eletromagnéticos (Olson e Bathe, 1985; Collop et al., 2003). Segundo Bettess (1992), o primeiro elemento infinito foi desenvolvido em 1973 por Ungless e Anderson, tendo este trabalho sido publicado apenas em 1977 (Anderson e Ungless, 1977). Este elemento foi utilizado na solução do problema de carga pontual aplicada em um semi-espaço infinito, solucionado analiticamente por Boussinesq, obtendo boa concordância com a solução exata.

Desde então, com o surgimento de computadores mais potentes e a consequente possibilidade de sofisticação das análises computacionais, novos elementos infinitos unidimensionais, bidimensionais e tridimensionais (Marques e Owen, 1984) foram desenvolvidos. A formulação para simulação do infinito consiste em usar funções de mapeamento assintóticas ou funções de forma com decaimento (Lynn e Hadid, 1983). Os primeiros resultados de elementos infinitos formulados a partir de funções assintóticas de mapeamento foram publicados por Beer e Meek (1981) em análise de tensões e deslocamentos gerados por escavação em meio pré-tensionado.

Em 1997, Hjelmstad et al. adotaram elementos infinitos para realizar análises elásticas de pavimentos rígidos. Recentemente, Al-Qadi et al. (2010) utilizaram este tipo de elemento para realizar análises dinâmicas não lineares de pavimentos, enquanto Rahman et al. (2010) realizaram análises de pavimentos asfálticos considerando diferentes tipos de eixos, área de contato e pressão do pneu. Nos dois últimos casos, os autores utilizaram o sistema computacional ABAQUS.

Este trabalho utiliza elementos infinitos com mapeamento assintótico porque estes são de formulação matemática e implementação computacional mais simples. A Figura 2 (a e b) mostra um elemento infinito unidimensional que, apesar de não ter aplicação prática no presente trabalho, é importante para a compreensão da ideia dos elementos infinitos mapeados.
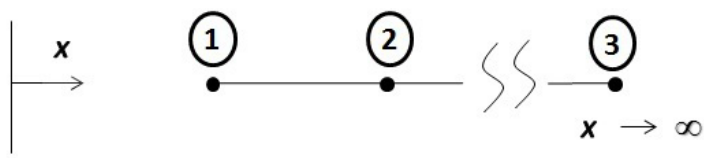

(a) 1D - Coordenadas cartesianas

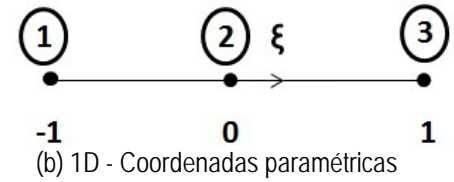

Figura 2. Elemento infinito unidimensional (1D)
A geometria deste elemento é dada pela função

$$
x=M_{1} x_{1}+M_{2} x_{2} \quad \text { com } M_{1}=-\frac{2 \xi}{1-\xi} \text { e } M_{2}=\frac{1+\xi}{1-\xi}
$$

em que $x_{1}$ e $x_{2}$ são as coordenadas cartesianas dos nós 1 e 2 , respectivamente (Figura $2 \mathrm{a}$ ). Pode-se verificar que $x(\xi=-1)$ $=x_{1}, x(\xi=0)=x_{2}$ e que quando a coordenada $\xi$ se aproxima de 1 , a coordenada $x$ tende ao infinito.

Como os deslocamentos (u) no infinito são nulos, então $\mathrm{u}_{3}=0$ e os deslocamentos no interior do elemento infinito são interpolados utilizando a expressão:

$$
u=N_{1} u_{1}+N_{2} u_{2}
$$

As funções de interpolação dos deslocamentos $N_{i}$ são os polinômios de Lagrange quadráticos convencionais (Cook et al., 2002). Utilizando-se estas funções de interpolação, considerando-se que o polo está na origem das coordenadas e que $x_{1}=a$ e $x_{2}=2 a$ pode-se mostrar que:

$$
\xi=1-\frac{2 a}{x} \text { e } u=\left(-u_{1}+4 u_{2}\right) \frac{a}{x}+\left(2 u_{1}-4 u_{2}\right) \frac{a^{2}}{x^{2}}
$$

Estas expressões mostram que o deslocamento $u$ tende a 0 quando $x$ vai para o infinito. Os deslocamentos no polo seriam infinitos, mas este ponto não faz parte do elemento e pode ser interpretado apenas como o ponto a partir de onde os deslocamentos decaem, como centro da região carregada. É importante notar que as coordenadas e os deslocamentos do nó 3 não aparecem nas Equações (7) e (8), portanto os nós do infinito não precisam ser considerados na geração da malha.

\subsection{Elemento infinito de Lagrange de 6 (seis) nós}

Neste trabalho foi adotado o elemento infinito bidimensional Lagrangeano de 6 nós, aqui denominado L6, ilustrado na Figura 3. Este elemento pode ser visto como um elemento finito Lagrangeano de 9 nós (Q9) com os 3 nós associados a $\eta=1$ posicionados no infinito (Marques e Owen, 1984). Desta forma, o elemento possui funções de mapeamento que tendem ao infinito à medida que a coordenada local $\eta$ tende a 1 , o mesmo não ocorrendo com a coordenada local ortogonal $\xi$, pois este elemento tende ao infinito em apenas uma direção.

As funções nodais de mapeamento $(M)$ e de forma $(N)$ deste elemento estão apresentadas na Tabela 1 (Marques e

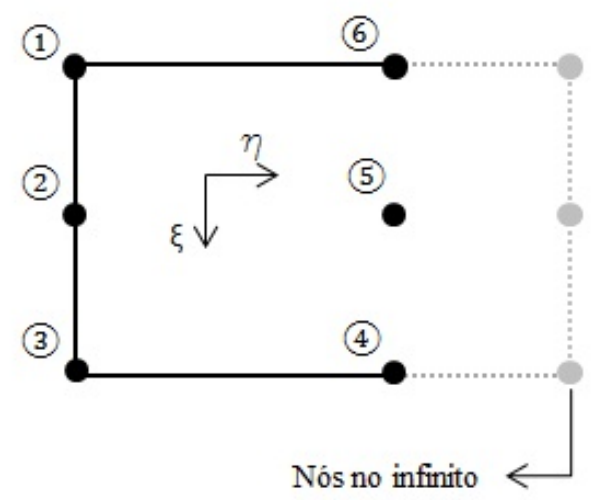

Figura 3. Elemento infinito bidimensional (2D) Lagrangeano de 6 nós 
Tabela 1. Funções de Mapeamento e de Forma do L6

\begin{tabular}{ll}
\hline Funções de Mapeamento & Funções de Forma \\
\hline$M 1=\frac{\left(\xi^{2}-\xi\right)(-\eta)}{(1-\eta)}$ & $N 1=\frac{\left(\xi^{2}-\xi\right)\left(\eta^{2}-\eta\right)}{4}$ \\
\hline $2=\frac{\left(1-\xi^{2}\right)(-2 \eta)}{(1-\eta)}$ & $N 2=\frac{\left(1-\xi^{2}\right)\left(\eta^{2}-\eta\right)}{2}$ \\
$M 3=\frac{\left(\xi^{2}+\xi\right)(-\eta)}{(1-\eta)}$ & $N 3=\frac{\left(\xi^{2}+\xi\right)\left(\eta^{2}-\eta\right)}{4}$ \\
$M 4=\frac{\left(\xi^{2}+\xi\right)(1+\eta)}{2(1-\eta)}$ & $N 4=\frac{\left(\xi^{2}+\xi\right)\left(1-\eta^{2}\right)}{2}$ \\
$M 5=\frac{\left(1-\xi^{2}\right)(1+\eta)}{(1-\eta)}$ & $N 5=\left(1-\xi^{2}\right)\left(1-\eta^{2}\right)$ \\
$M 6=\frac{\left(\xi^{2}-\xi\right)(1+\eta)}{2(1-\eta)}$ & $N 6=\frac{\left(\xi^{2}-\xi\right)\left(1-\eta^{2}\right)}{2}$ \\
\hline
\end{tabular}

Owen, 1984).

A implementação deste elemento foi realizada no CAP3D que é um programa para análise de pavimentos através do MEF desenvolvido no Laboratório de Mecânica dos Pavimentos da Universidade Federal do Ceará (Holanda et al., 2006). No seu estágio atual, o CAP3D é capaz de tratar modelos planos, axissimétricos e tridimensionais, utilizando elementos de diferentes formas e ordens de interpolação (linear e quadrática). O sistema ainda é capaz de realizar análises lineares e não lineares, estáticas e dinâmicas (Araújo et al., 2010).

\section{GERAÇÃO DE MALHA}

A geração de malha para aplicação do Método dos Elementos Finitos (MEF) foi realizada em um pré-processador, o Pavement Mesh Generator (PMG), ilustrado na Figura 4, também desenvolvido no LMP/UFC (Coutinho et al.; 2008; Cavalcante et al., 2008; Botelho et al., 2009).

Este sistema foi desenvolvido especificamente para análise de pavimentos e tem por função receber os dados de entrada do problema, como raio e módulo de carregamento, espessura das camadas e propriedades dos materiais, e gerar

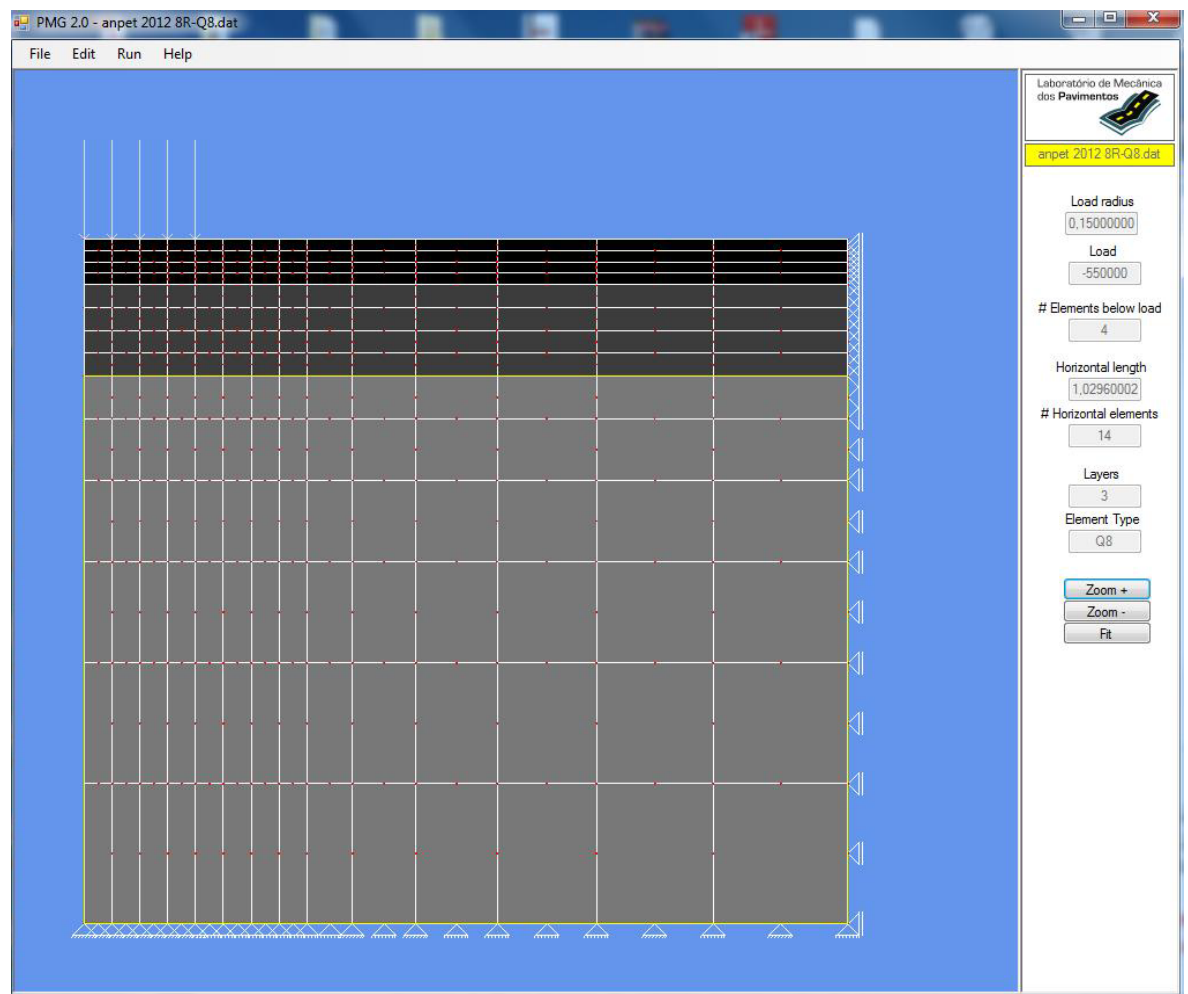

Figura 4. Sistema Computacional PMG 


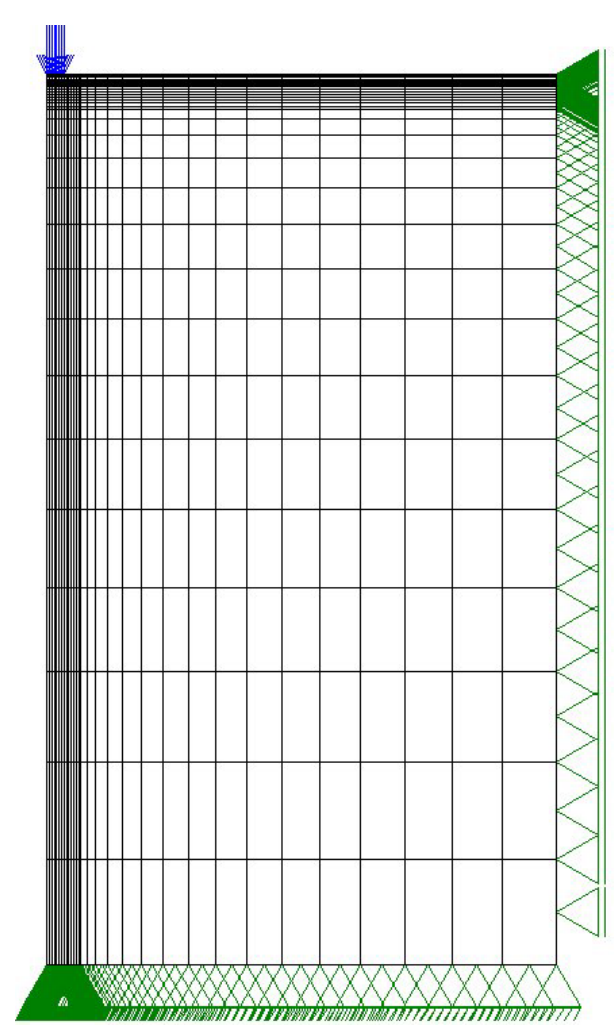

(a) Elementos Finitos

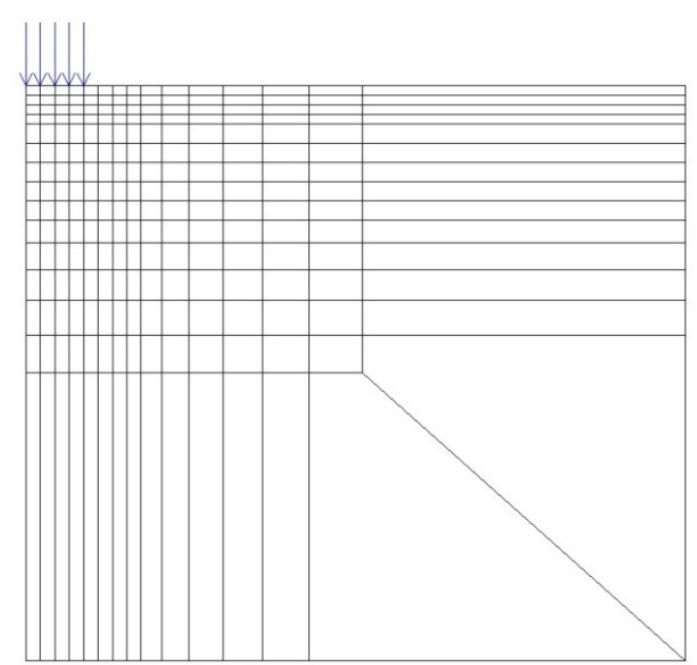

(b) Elementos infinitos e finitos

Figura 5. Geração da malha

uma malha de elementos finitos. Após a geração da malha o PMG executa a análise automaticamente utilizando o programa CAP3D.

Para a malha de elementos finitos utilizou-se o elemento quadrático de 8 (oito) nós (Q8), e, seguindo as orientações presentes na literatura (NCHRP/TRB, 2004) foi gerada uma malha com o comprimento vertical igual a 50 (cinquenta) vezes o raio de aplicação da carga, e comprimento horizontal equivalente a 12 vezes o comprimento deste mesmo raio (Figura 5a).

Tais imposições têm o propósito de simular o infinito e evitar que o truncamento dos deslocamentos e tensões gerado pela proximidade do apoio à carga leve a resultados incorretos. Porém, esta abordagem faz com que a malha tenha um número excessivo de elementos e nós, o que resulta no aumento do número de incógnitas, aumentando por consequência o tempo de processamento da análise.

Implementou-se no PMG uma rotina de geração de malhas mistas de elementos finitos (Q8) e infinitos (L6). A Figura $5 \mathrm{~b}$ mostra um exemplo desta malha mista. Observa-se que não são mais necessários apoios no contorno do modelo, pois quando a função de mapeamento tende ao infinito, o deslocamento tende a zero sem a necessidade da restrição de movimento imposta.

O polo está localizado na superfície e sobre o eixo do modelo axissimétrico (canto superior esquerdo da Figura 5b). Assim, de maneira a garantir a qualidade dos resultados, a malha é gerada pelo PMG de forma que a distância entre os nós do elemento infinito sejam iguais à distância entre os eixos cartesianos que passam pelo polo e o início do elemento infinito, como mostrado de forma esquemática pela Figura 6. Esta forma de geração de malha combina uma boa discretização em elementos finitos na região de interesse próxima ao carregamento, onde as tensões e deformações são maiores, com elementos infinitos para represen- tar o solo circundante ao pavimento.

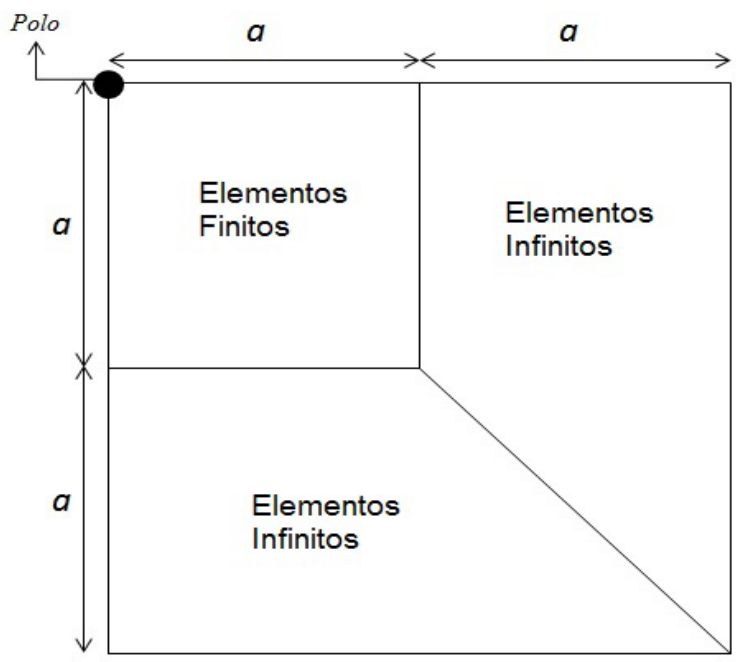

Figura 6. Localização do polo

\section{EXEMPLOS NUMÉRICOS}

\subsection{Validação da malha de elementos finitos e da malha mista}

O objetivo deste exemplo é avaliar a qualidade dos resultados obtidos ao se adotar uma malha mista com uma menor quantidade de elementos. Realizou-se inicialmente uma análise elástico-linear comparativa entre a malha de elementos finitos e a malha mista, ambas com profundidade equivalente a 50 vezes o raio de aplicação do carregamento. Os resultados foram comparados com os obtidos pelo programa SisPav (Franco, 2007), que utiliza o Método da Teoria de 
Tabela 2. Estrutura de Pavimentos

\begin{tabular}{llll}
\hline & Espessura(m) & Módulo de Elasticidade(MPa) & Poisson \\
\hline Revestimento & 0,10 & 3500 & 0,35 \\
Base & 0,20 & 350 & 0,30 \\
Subleito & 7,50 & 100 & 0,40 \\
\hline
\end{tabular}

Tabela 3. Deslocamentos verticais $(\mathrm{mm})$

\begin{tabular}{cllcll}
\hline $\mathbf{Z}(\mathbf{m m})$ & SisPav/AEMC & $\begin{array}{l}\text { PMG/CAP3D } \\
(\mathbf{M M}-\mathbf{Q 8} / \mathbf{L} \text { ) }\end{array}$ & Diferença & $\begin{array}{c}\text { PMG/CAP3D } \\
(\mathbf{M E F}-\mathbf{Q 8})\end{array}$ & Diferença \\
\hline 0 & 0,448 & 0,448 & $0,0 \%$ & 0,420 & $-6,4 \%$ \\
\hline 50 & 0,451 & 0,450 & $-0,1 \%$ & 0,422 & $-6,3 \%$ \\
\hline 100 & 0,442 & 0,442 & $0,0 \%$ & 0,414 & $-6,4 \%$ \\
\hline 200 & 0,387 & 0,386 & $-0,3 \%$ & 0,359 & $-7,2 \%$ \\
\hline 300 & 0,350 & 0,350 & $0,0 \%$ & 0,322 & $-8,0 \%$ \\
\hline
\end{tabular}

Múltiplas Camadas Elásticas (TMCE). Foi aplicada nesta análise uma carga de $550 \mathrm{kPa}$ em um raio de $0,15 \mathrm{~m}$. A estrutura solicitada (NCHRP/TRB, 2004) se constituía de revestimento asfáltico, base em solo brita (crushed stone base) e subleito com baixa resistência (soft subgrade), cujos parâmetros físicos constam na Tabela 2.

A Tabela 3 mostra os resultados de deslocamentos verticais obtidos pelo SisPav, bem como os resultados obtidos pelo uso do PMG/CAP3D com a malha contendo apenas elementos finitos (MEF-Q8) e a malha mista, composta por elementos finitos e infinitos (MM-Q8/L6). Os valores obtidos pelo programa SisPav foram tomados como referência em função da TMCE fornecer resultados exatos para este caso. Assim, as diferenças percentuais apresentadas na Tabela 3 são calculadas sempre entre o PMG/CAP3D e o SisPav.

Pode-se perceber que os resultados em deslocamentos apresentados pelo programa SisPav e pelo PMG/CAP3D com o uso da malha mista foram praticamente idênticos, enquanto a estrutura modelada apenas com elementos finitos apresentou deslocamentos menores que os demais, apresentando, portanto, comportamento mais rígido.

Esta maior rigidez pode ser explicada pela distância dos nós da extremidade inferior, onde os deslocamentos são nulos, em relação ao topo do pavimento. Enquanto esta distância é finita para a malha contendo apenas elementos finitos, os nós desta extremidade na malha mista são posiciona- dos geometricamente no infinito, representando melhor o comportamento do subleito.

Desta forma, os apoios da estrutura de elementos finitos, que estão mais próximos do topo do pavimento, impõem deslocamento nulo de maneira mais brusca que nos elementos infinitos. Por outro lado, no caso das tensões verticais (Tabela 4) e horizontais (Tabela 5) tanto a malha de elementos finitos como a malha mista levaram a valores praticamente iguais aos obtidos pela TMCE (SisPav).

\subsection{Teste de convergência}

Admitindo-se que os resultados da malha mista anterior atingiram a convergência, realizaram-se diversas análises, a fim de verificar a partir de que relação entre o raio de aplicação da carga (R) e o comprimento vertical do modelo se daria a convergência dos resultados, comparando-se estes com o obtido pela rotina AEMC do SisPav. A Tabela 6 apresenta as diferenças numéricas encontradas para as variáveis de maior interesse do método mecanístico-empírico de dimensionamento: deslocamento no topo do revestimento, tensão horizontal no fundo do revestimento e tensão vertical no topo do subleito.

Verifica-se que os erros são desprezíveis do ponto de vista prático para todas as malhas consideradas. Por outro lado, em termos de desempenho a malha com comprimento vertical igual a 50R gerou um modelo de 2913 nós e seu tempo de análise foi de 0,670s, enquanto a malha de com-

Tabela 4. Tensões Verticais $(\mathrm{kPa})$

\begin{tabular}{ccclcl}
\hline & & PMG/CAP3D & \multicolumn{3}{c}{ PMG/CAP3D } \\
$\mathbf{Z}(\mathbf{m m})$ & SisPav/AEMC & (MM - Q8/L6) & Diferença & (MEF - Q8) & Diferença \\
\hline 0 & $-550,000$ & $-552,074$ & $-0,4 \%$ & $-552,074$ & $-0,4 \%$ \\
100 & $-248,136$ & $-246,293$ & $-0,7 \%$ & $-246,293$ & $-0,7 \%$ \\
300 & $-63,580$ & $-63,176$ & $-0,6 \%$ & $-63,179$ & $-0,6 \%$ \\
\hline
\end{tabular}

Tabela 5. Tensões Horizontais $(\mathrm{kPa})$

\begin{tabular}{|c|c|c|c|c|c|}
\hline$Z(\mathrm{~mm})$ & SisPav/AEMC & $\begin{array}{l}P M G / C A P 3 D \\
(M M-Q 8 / L 6)\end{array}$ & Diferença & $\begin{array}{l}\text { PMG/CAP3D } \\
\text { (MEF - Q8) }\end{array}$ & Diferença \\
\hline 0 & $-1609,100$ & $-1613,250$ & $-0,3 \%$ & $-1611,09$ & $-0,1 \%$ \\
\hline 100 & 1008,156 & 1012,400 & $-0,4 \%$ & 1014,09 & $-0,6 \%$ \\
\hline 300 & 100,707 & 100,615 & $0,1 \%$ & 100,664 & $0,0 \%$ \\
\hline
\end{tabular}

Tabela 6. Diferenças percentuais - PMG/CAP3D - SisPav/AEMC

\begin{tabular}{|c|c|c|c|c|c|c|c|}
\hline & $8 R$ & $10 R$ & $12 R$ & $14 R$ & $16 R$ & $18 R$ & $20 R$ \\
\hline Deflexão (mm) & $-0,4 \%$ & $-0,4 \%$ & $-0,2 \%$ & $-0,2 \%$ & $-0,2 \%$ & $-0,2 \%$ & $-0,2 \%$ \\
\hline Tensão horizontal (kPa) & $0,6 \%$ & $0,6 \%$ & $0,6 \%$ & $0,6 \%$ & $0,6 \%$ & $0,6 \%$ & $0,6 \%$ \\
\hline Tensão vertical (kPa) & $0,0 \%$ & $-0,3 \%$ & $-0,1 \%$ & $-0,2 \%$ & $-0,4 \%$ & $-0,4 \%$ & $-0,4 \%$ \\
\hline
\end{tabular}


primento vertical igual a $10 \mathrm{R}$ resultou em um modelo de apenas 702 nós e levou 0,093s para ser analisada, aproximadamente sete vezes menos que a malha mais refinada.

\subsection{Pavimento com revestimento viscoelástico}

Este exemplo tem a finalidade de avaliar o desempenho da análise utilizando a malha mista em termos de qualidade de resposta e tempo de execução, considerando o revestimento asfáltico como viscoelástico linear. As mesmas características geométricas do pavimento do exemplo anterior foram adotadas, assim como o mesmo material da base e subleito. O raio de aplicação e o módulo da carga também são mantidos, sendo que a carga tem a forma de um pulso semisenoidal de $0,01 \mathrm{~s}$.

A série de Prony utilizada para representar o comportamento mecânico do revestimento asfáltico é descrita na Tabela 7 (Lee, 1996). Foi mantido o coeficiente de Poisson de 0,35 para o revestimento. Portela et. al (2008) determinaram computacionalmente um Módulo de Resiliência (MR) equivalente para a mistura considerando esta série de Prony e um pulso semi-senoidal de 0,01s, obtendo um MR de 4838,08 MPa.

Os valores de deflexão (Figura 7) obtidos quando a malha de EF foi adotada foram, no intervalo de aplicação da carga, em torno de $6 \%$ menores que os obtidos com a malha mista. Conforme observado no item 5.1, o uso de malhas contendo apenas elementos finitos tende a subestimar os deslocamentos do pavimento. O comportamento da curva considerando o Módulo de Resiliência (MR) igual a 4838,08 MPa ratificou que este é o MR equivalente da mistura. É importante ressaltar que a análise elástica foi realizada utilizando a malha mista. Nas Figuras 7, 8 e 9, a variável $t_{c}$ representa o tempo de aplicação do carregamento.
Tabela 7. Série de Prony

\begin{tabular}{lll}
\hline Termo & $\boldsymbol{E}(\mathbf{k P a})$ & $\boldsymbol{\rho}(\mathbf{s})$ \\
\hline$\infty$ & $1,172 \mathrm{E}+03$ & - \\
\hline 1 & $3,10 \mathrm{E}+06$ & $2,20 \mathrm{E}-05$ \\
\hline 2 & $4,31 \mathrm{E}+06$ & $2,20 \mathrm{E}-04$ \\
\hline 3 & $3,46 \mathrm{E}+06$ & $2,20 \mathrm{E}-03$ \\
\hline 4 & $2,02 \mathrm{E}+06$ & $2,20 \mathrm{E}-02$ \\
\hline 5 & $1,27 \mathrm{E}+06$ & $2,20 \mathrm{E}-01$ \\
\hline 6 & $2,72 \mathrm{E}+05$ & $2,20 \mathrm{E}+00$ \\
\hline 7 & $6,59 \mathrm{E}+04$ & $2,20 \mathrm{E}+01$ \\
\hline 8 & $1,45 \mathrm{E}+04$ & $2,20 \mathrm{E}+02$ \\
\hline 9 & $1,52 \mathrm{E}+03$ & $2,20 \mathrm{E}+03$ \\
\hline 10 & $7,10 \mathrm{E}+02$ & $2,20 \mathrm{E}+04$ \\
\hline 11 & $5,88 \mathrm{E}+01$ & $2,20 \mathrm{E}+05$ \\
\hline
\end{tabular}

Os resultados de tensões horizontais no fundo do revestimento (Figura 8) para as duas malhas foram bastante próximos, assim como observado no item 5.1, mostrando que a modificação na malha não trouxe diferenças consideráveis nos valores desta variável. A consideração da viscoelasticidade neste caso é importante, pois se pode verificar a inversão do sentido das tensões normais, o que agrava o efeito da fadiga no revestimento asfáltico.

A Figura 9 apresenta a tensão vertical no topo do subleito, onde se verifica que não há grandes modificações nos valores máximos ao se utilizar as diferentes malhas. Pode-se ainda notar que a consideração do revestimento como material viscoelástico pouco afetou a tensão vertical máxima, porém, percebe-se que a estrutura acumula tensões mesmo depois de cessada a aplicação de carga neste modelo, o que pode se tornar cumulativo se forem aplicados carregamentos sucessivos, solicitando mais fortemente a estrutura.

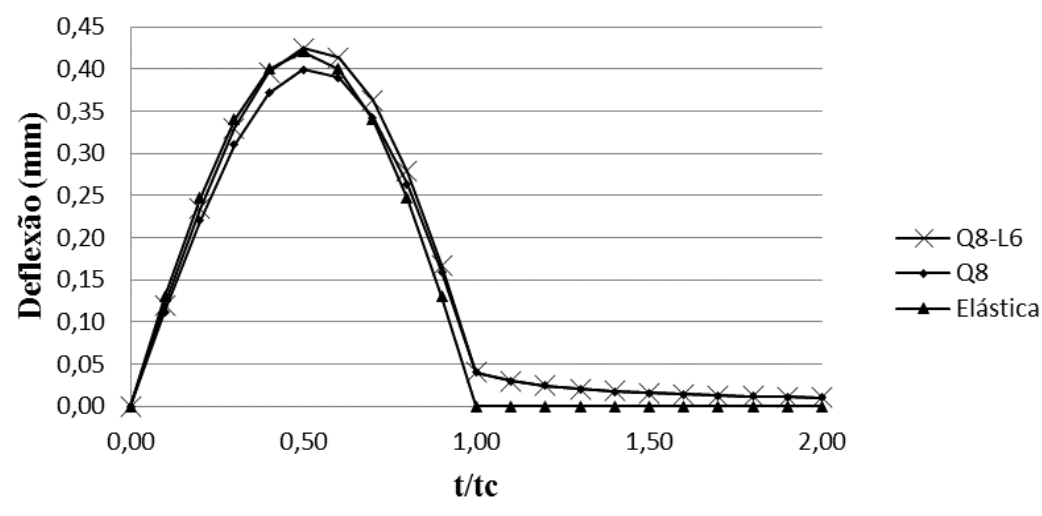

Figura 7. Deflexões da malha mista e da malha de elementos finitos

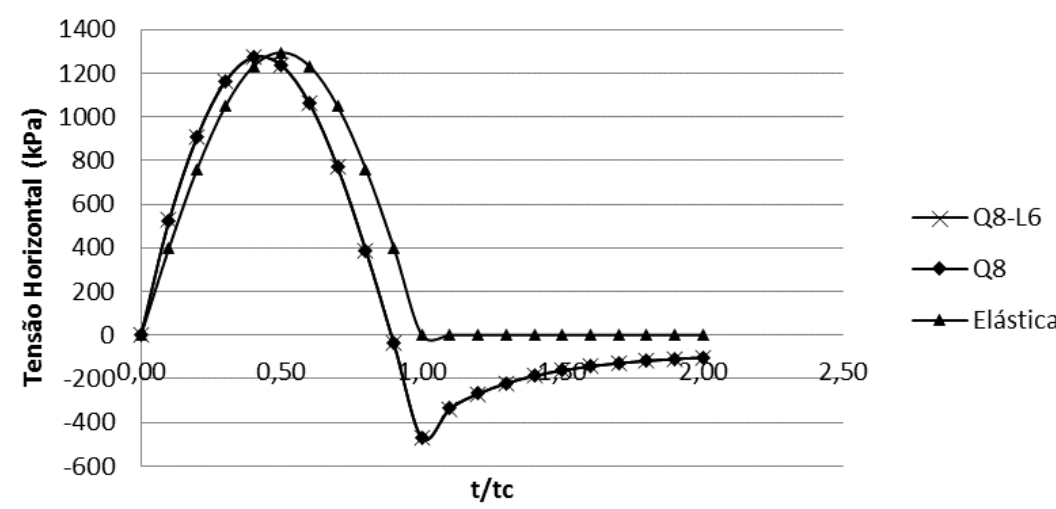

Figura 8. Tensão horizontal no fundo do revestimento 


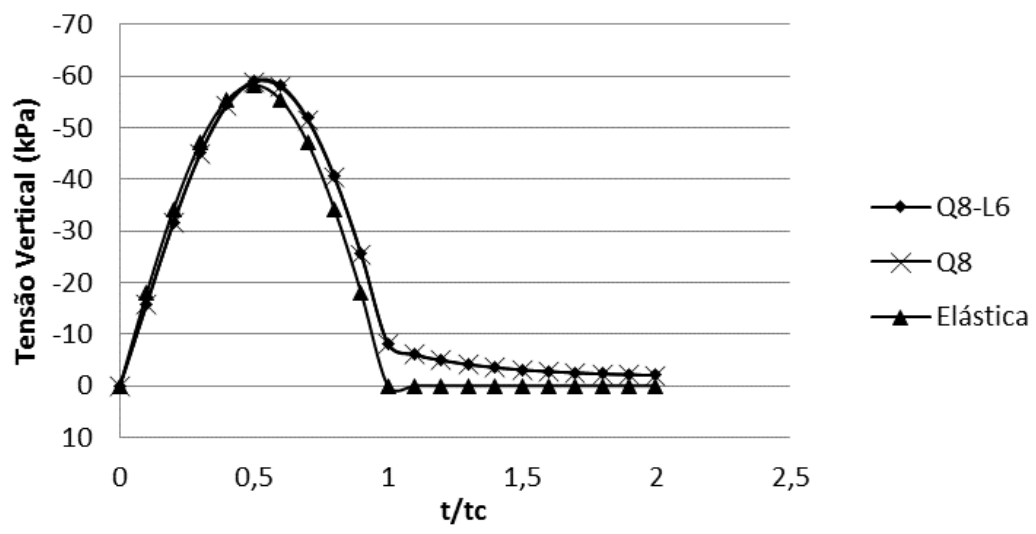

Figura 9. Tensão vertical no topo do subleito

Assim como no exemplo anterior, a análise da malha gerada com elementos finitos e infinitos apresentou um tempo de análise bastante reduzido em relação à análise da malha de elementos finitos. Enquanto a primeira foi analisada em 2,48s, a segunda levou 11,69s para ser analisada. Assim, a análise com malha mista foi 4,7 vezes mais eficiente que a com malha convencional. A maior duração e diferença do tempo de análise em relação aos exemplos anteriores ocorrem devido ao método incremental utilizado ao se considerar o material de revestimento viscoelástico (análise dependente do tempo), diferente dos exemplos anteriores, nos quais todas as camadas foram consideradas elásticas.

É importante ressaltar que a redução do tempo de processamento obtida nos exemplos apresentados neste trabalho pode não parecer significativa ao se considerar uma análise isolada, considerando que o tempo total de análise é pequeno da ordem de segundos. Contudo, para a realização de retro-análises e de projeto utilizando algoritmos de otimização, é necessário realizar um elevado número de análises até que o processo seja finalizado. Nestes casos, a aplicação da metodologia proposta representa uma significativa redução no tempo de análise. Adicionalmente, os resultados obtidos em 2D neste trabalho indicam que os ganhos de tempo em problemas 3D, onde o tempo de processamento de modelos de elementos finitos para análise elástica linear é da ordem de minutos, podem ser bastante significativos.

\section{CONSIDERAÇÕES FINAIS}

Este trabalho apresentou uma estratégia para aumento da precisão e eficiência da análise de pavimentos flexíveis baseada no uso de elementos infinitos. Uma breve revisão do modelo viscoelástico linear foi realizada, além de uma breve introdução aos elementos infinitos, com ênfase para os elementos infinitos com mapeamento assintótico. A geração da malha mista (elementos finitos e infinitos) no PMG foi descrita e exemplos numéricos foram realizados.

Em todas as análises realizadas, a malha mista foi mais eficiente do ponto de vista computacional, o que já era esperado em função da diminuição do número de nós e elementos. Apesar do tempo das análises bidimensionais de pavimentos não ser muito elevado, o mesmo não ocorre no caso de análises tridimensionais, onde a necessidade de memória e tempo de processamento são consideravelmente maiores.

Para o caso de análises dependentes do tempo ou nãolineares, a diminuição do tempo de processamento que o uso da malha mista proporciona tende a ser ainda maior, pois para esses tipos de análise são utilizados métodos iterativos nos quais várias análises internas são realizadas para chegar-se ao resultado final. Além da maior eficiência computacional, o uso da malha mista apresentou resultados mais precisos do que os obtidos com o uso da malha contendo apenas elementos finitos em todos os exemplos apresentados.

O tempo de processamento na análise viscoelástica foi significantemente maior que o da análise elástica para a mesma malha, o que também era previsto, pois a análise viscoelástica é realizada através de algoritmo incremental utilizando vários passos de tempo. O ganho de eficiência na análise viscoelástica foi um pouco inferior ao registrado na análise elástica, mas mesmo assim foi bastante significativo com redução de 4,7 vezes no tempo de processamento.

Assim, pode-se concluir que o uso de malhas mistas é bastante recomendado para análise de pavimentos flexíveis. Esta abordagem permite aumentar a precisão e eficiência da análise por elementos finitos, reduzindo a diferença de tempo em relação ao uso da Teoria da Múltiplas Camadas Elásticas em ampla utilização atualmente, com a vantagem de permitir a consideração de modelos constitutivos não lineares ou dependentes do tempo.

\section{AGRADECIMENTOS}

Os autores agradecem ao CNPq, FINEP, PETROBRAS e UFC pelo apoio financeiro a esta pesquisa.

\section{REFERÊNCIAS BIBLIOGRÁFICAS}

Al-Qadi, I. L.; H. Wang e E. Tutumluer (2010) Dynamic Analysis of Thin Asphalt Pavements by Using Cross-Anisotropic Stress-Dependent Properties for Granular Layer. Journal of the Transportation Research Board. n. 2154, p. 156-163. DOI: 10.3141/2154-16.

Anderson, D. L. e R. L. Ungless (1977) Infinite Elements. International Symposium of Innovative Numerical and Analytical Applied Engineering Science. França.

Araújo, P. C.; J. B. Soares; A. S. Holanda; E. P. Parente e F. Evangelista (2010) Dynamic Viscoelastic Analysis of Asphalt Pavements using Finite Element Formulation. Road Materials and Pavement Design, v. 11, p. 409-433.

Beer, G. e J. L. Meek (1981) Infinite Domain Elements. International Journal of Numerical Methods in Engineering, v. 17, n. 1, p. 4352.

Bernucci, L. B.; L. M. G. Motta; J. A. P. Ceratti e J. B. Soares (2007) Pavimentação Asfáltica, Second edition, Petrobras: ABEDA, Rio de Janeiro, RJ.

Bettess, P. (1992) Infinite Elements. Ed. Penshaw Press, Inglaterra.

Botelho, R. P.; R. N. A. Cavalcante; R. P. Coutinho; A. S. Holanda e E. Parente Jr (2009) Desenvolvimento de um Sistema Gráfico para 
Análise Numérica de Pavimentos Asfálticos, IV Simpósio Internacional de Avaliação de Pavimentos e Projetos de Reforço, Fortaleza, CE, p. 1-12.

Cavalcante, R. N. A.; R. P. Coutinho; A. S. Holanda e E. Parente Jr (2008). Gerador de Malha Bidimensional para Análise de Tensões e Deformações em Pavimentos Asfálticos. Anais do XXIX Iberian Latin American Congress on Computational Methods in Engineering, CILAMCE, p. 1-18.

Christensen, R. M. (1982) Theory of Viscoelasticity. Dover (2a ed.)., New York, NY, USA.

Collop, A. C.; A. T. Scarpas; C. Kasbergen e A. de Bondt (2003). Development and Finite Implementation of a Stress Dependent ElastoVisco-Plastic Constitutive Model with Damage for Asphalt. Transportation Research Board 82nd, Annual Meeting. p. 1-27.

Cook, R.; D. Malkus e M. Plesha (2002) Concepts and Applications of Finite Element Analysis. 2a Edição, Editora John Wiley \& Sons.

Coutinho R. P.; R. N. A. Cavalcante; A. S. Holanda e E. Parente Jr (2008). Desenvolvimento de um Gerador de Malhas para Análise de Pavimentos Pelo Método dos Elementos Finitos. Anais do XXII Congresso de Pesquisa e Ensino em Transportes, ANPET, Fortaleza - CE, p. 1-12

Davids, W. G. (2009) EverStress FE1.0 Software for 3D Finite Element Analysis of Flexible Pavement Structures: Summary of Features and Capabilities, p. 1-4.

Franco, F. A. C. P. (2007) Método de dimensionamento mecanísticoempírico de pavimentos asfálticos - SISPAV. Tese (Doutorado em Engenharia Civil), Universidade Federal do Rio de Janeiro.

Hjelmstad, K. D.; Q. Zuo e J. Kim (1997). Elastic Pavement Analysis Using Infinite Elements. Transportation Research Record: Journal of the Transportation Research Board. v. 1568, p. 72-76. DOI: 10.3141/1568-09.

Holanda, A. S.; E. Parente Jr.; T. D. P. Araújo; L. T. B. Melo; F. Evangelista Jr. e J. B. Soares (2006). Finite Element Modeling of Flexible Pavements. XXVII Iberian Latin-American Congress on Computational Methods in Engineering (CILAMCE), Belém, Pará.

Huang, Y. H. (2004) Pavement Analysis and Design, Second edition, Prentice Hall, Inc., Englewood Cliffs, New Jersey, USA.

Lakes, R. S. (1998) Viscoelastic Solids. CRC Press.

Lee, H. J. (1996). Uniaxial Constitutive Modeling of Asphalt Concrete Using Viscoelasticity and Continuum Damage Modeling. PhD thesis, Civil Engineering Department, North Carolina University, USA.

Lynn, P. P. e Hadid, H. A. (1981) Infinite Elements with 1/rn type decay. International Journal of Numerical Methods in Engineering, v. 17, n. 3, p. 347-355.

Marques, J. M. M. C. e D. R. J. Owen (1984) Infinite Elements in Quasistatic Materially Nonlinear Problems. Computers and Structures, v. 18 , n. 4 , p. $739-751$.

Medina, J. e L. M. G. Mota (2005). Mecânica dos Pavimentos. 2a edição. 574p. Editora UFRJ. Rio de Janeiro. RJ.

Motta, L. M. G. (1991) Método de Dimensionamento de Pavimentos Fle xíveis; Critério de Confiabilidade e Ensaios de Cargas Repetidas. Tese de D.Sc., COPPE/UFRJ, Rio de Janeiro, RJ, Brasil.

NCHRP/TRB (2004) Guide for Mechanistic-Empirical Design of New and Rehabilitated Pavement Structures, Appendix RR: Finite Element Procedures for Flexible Pavement Analysis, Illinois, USA.

Olson, L. G. e K. J. Bathe (1985). An Infinite Element for Analysis of Transient Fluid-Structure Interactions. Eng. Comput., v. 2, p. 319-329.

Portela, E. L.; E. Parente Jr.; R. P. Botelho e A. S. Holanda (2008) Análise Viscoelástica de Pavimentos Asfálticos Pelo Método dos Elementos Finitos. Anais do XXII Congresso de Pesquisa e Ensino em Transportes, ANPET, Fortaleza - CE, p. 1-12.

Rahman, M. T.; K. Mahmud e S. Ahsan (2011). Non Stress-Strain Characteristics of Flexible Pavement Using Finite Element Analysis. Int. Journal of Civil and Structural Engineering, v. 2, n. 1, p. 233-249.

Shames, I. H. e F. A. Cozzarelli (1997) Elastic and Inelastic Stress Analysis. Taylor \& Francis, Revised Printing.

Simulia (2007) ABAQUS User's Manual, Version 6.7.

Soares, J. B.; A. Mateos e L.M.G. Motta (2009) Aspectos gerais de métodos de dimensionamento de pavimentos asfálticos de vários países e a relação com um novo método brasileiro. Revista Pavimentação (ABPv), Outubro, p. 20-35.

Soares, J. B. e F. Souza (2002) Verificação do Comportamento Viscoelástico Linear em Misturas Asfálticas. Anais do $16^{\circ}$ Encontro de Asfalto, IBP, Rio de Janeiro. 